\title{
Pollution urbaine en Afrique de l'Ouest
}

\author{
Cathy Liousse et Corinne Galy-Lacaux
}

Laboratoire d'aérologie, CNRS - Université de Toulouse, OMP

14, avenue Édouard-Belin - 31400 Toulouse

\section{Résumé}

Les pollutions urbaines et leurs impacts font l'objet de nombreuses études en Europe et en Amérique du Nord, où sont mises en œuvre des politiques de réduction des émissions, de combustion notamment. C'est encore loin d'être le cas en pays émergents et, surtout, dans les pays en voie de développement. L'Afrique de l'Ouest est tout particulièrement concernée. Nous présentons ici quelques résultats de travaux en cours, centrés sur la pollution de quelques capitales d'Afrique de l'Ouest, travaux qui se développent en collaboration avec des équipes européennes et africaines de physicochimie de l'atmosphère et de médecins. Ils cernent de façon saisissante l'importance de cette pollution.

\section{Abstract}

\section{Urban pollution in West Africa}

Urban pollution is investigated in the Northern Countries with regulation and laws to reduce their impact, this is not the case in developing countries and particularly in Africa. On-going studies are presented, focused on coupled pollution and health in a few West African capitals jointly developed by European and African physical chemists and medical researchers. They strongly highlight a very poor air quality as the source of severe health problems.
'Afrique est un intense émetteur anthropique (feux de biomasse, feux domestiques, trafic croissant, industries pétrolières et mines en plein essor), en conjonction avec un exode rural fort, une démographie galopante et une urbanisation explosive très supérieures à celles des pays développés, avec concentration des activités nationales (par exemple en termes de PNB) dans quelques villes. On s'attend dans une dizaine d'années à la formation de mégapoles en Afrique de l'Ouest (figure 1). Il en résultera une dégradation majeure de la qualité de l'air.

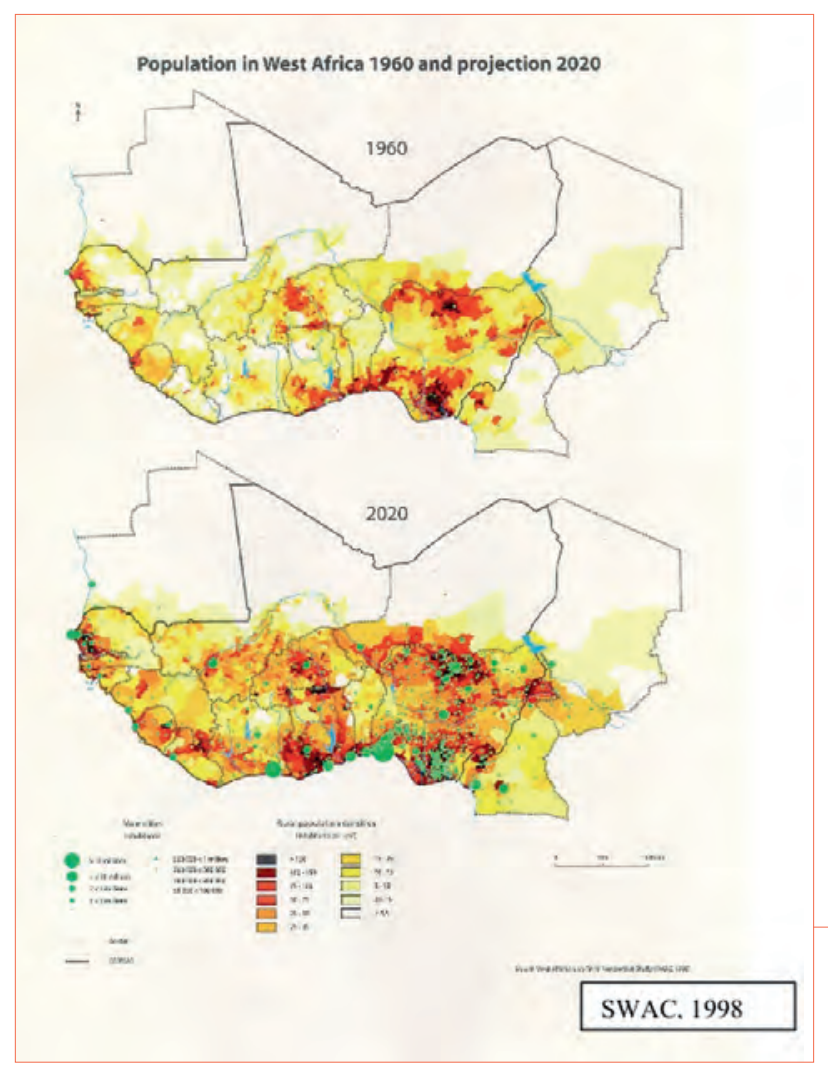

Depuis une vingtaine d'années, des programmes de recherche sont organisés sur le continent africain, par exemple :

- Decafe (Dynamique et chimie de 1'atmosphère en forêt équatoriale), 1986-1992;

- Expresso (Experiment for Regional Sources and Sinks of Oxidants), 19941998 ;

- Réseau de mesures Idaf (Igac/ Debits/Africa : International Global Atmospheric Chemistry/Deposition of Biogeochemical Important Trace Species/Africa), depuis 1994 ;

- Safari (Southern African Regional Science Initiative), 2000 ;

- Sacclap - Pics/ CNRS Afrique du Sud (Air Pollution and Climate Change in South Africa Programme international de coopération scientifique/Centre national de la recherche scientifique); - Amma (Analyse multidisciplinaire de la mousson africaine), 2005-2008.

Ces programmes ont particulièrement étudié les sources et puits des composés mineurs atmosphériques en milieu rural intertropical

Figure 1 - Prévisions de la formation de mégapoles en Afrique de l'Ouest à I'horizon 2020. (Source : OCDE, 1998). 
africain et leur impact sur 1'atmosphère à l'échelle régionale et continentale. Ce n'est que récemment que des études sont conduites sur la pollution d'origine anthropique dans les villes et mégapoles d'Afrique : Amma, Sacclap, Idaf, Polca (Pollution des capitales africaines).

\section{Un contexte favorable à la dégradation de la qualité de l'air}

La pollution dans les villes africaines apparaît suite à des émissions du même ordre de grandeur que les sources industrielles des pays du nord. Un séjour dans les grandes villes d'Afrique suffit pour réaliser l'importance de la pollution atmosphérique urbaine (photos ci-dessous, en plein cœur de Cotonou, capitale du Bénin). Cette pollution est amplifiée par les conditions climatiques (photochimie intense). Le suivi de la qualité de l'air dans les villes africaines est pratiquement inexistant et sans véritable texte réglementant les émissions de polluants. Certains efforts de lutte contre la pollution ont été initiés : c'est par exemple le cas du plomb avec des actions d'élimination de cette espèce dans l'essence des pays d'Afrique subsaharienne.

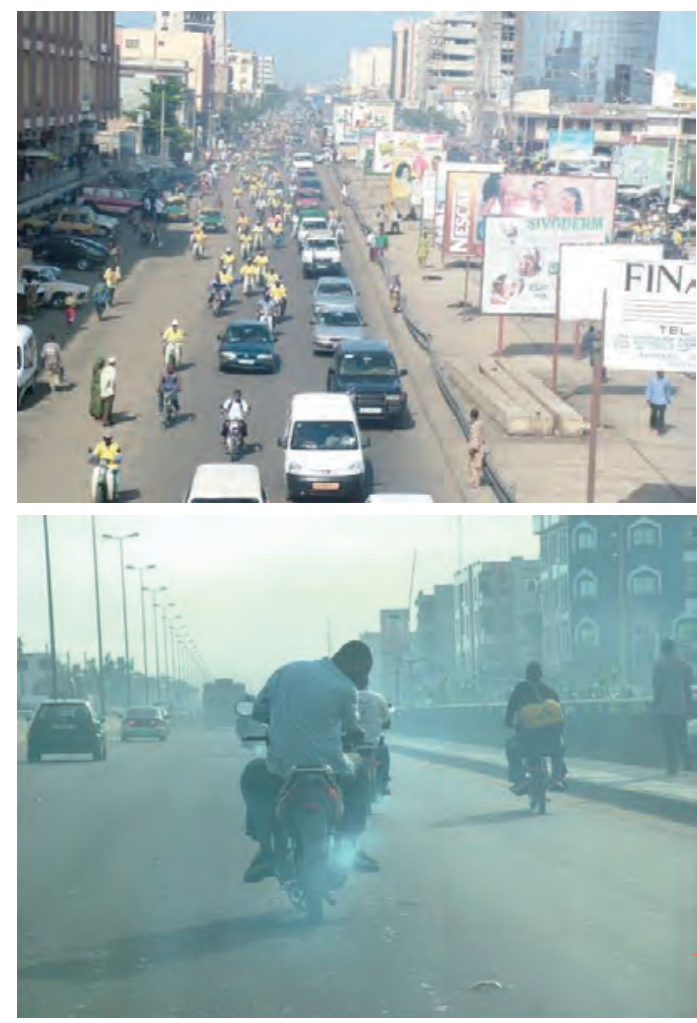

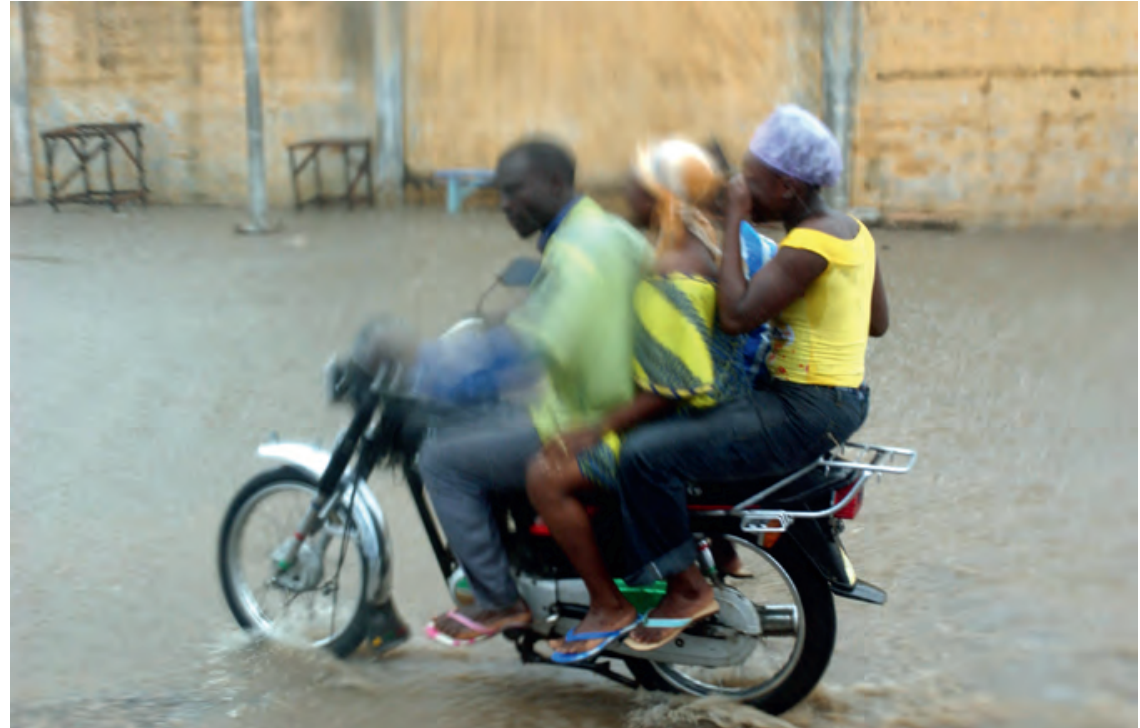

Rue au Bénin : pour des raisons économiques, il y a recrudescence du parc des deux-roues. (C) Météo-France Pascal Taburet)

Dans les capitales africaines, la pollution gazeuse et particulaire résulte principalement de la circulation automobile, des feux domestiques et, pour une moindre contribution, des émissions industrielles. Les émissions liées au trafic automobile sont responsables d'une pollution majeure. En effet, du fait de la réduction des transports publics et/ou de leur vétusté, il y a accroissement du parc automobile ; par ailleurs, le pouvoir d'achat étant faible, l'écrasante majorité de ces véhicules est de seconde main, pour la plupart importés d'Europe (essentiellement de France et de Belgique pour les pays francophones). L'âge de ces véhicules dépasse dix ans en moyenne sur l'Afrique de l'Ouest. Pour des raisons économiques encore, il y a recrudescence du parc des deuxroues avec moteurs deuxtemps, utilisant pour la plupart du fioul frelaté, moins cher et très polluant (photos ci-dessus et ci-contre). De plus, on peut noter que les infrastructures routières, les transports publics et l'aménagement urbain restent insuffisants, conduisant à d'importants ralentissements et congestions de la circulation qui aggravent le problème. Un inventaire d'émission récent montre que celles de particules organiques dues au trafic en Afrique de l'Ouest sont à multiplier par 10 à 30 si l'on tient compte des deux-roues (Assamoi et Liousse, 2010).

Rues de Cotonou au Bénin (campagne Amma 2005)
On comprend alors que les effets combinés de la croissance démographique, de l'urbanisation, de la motorisation et de l'utilisation de carburants de mauvaise qualité (avec encore des niveaux de plomb élevés) génèrent une pollution rapidement croissante dans les villes d'Afrique subsaharienne. Notons, par exemple, l'ampleur de l'exode rural. Il y a quarante ans, un Africain sur cinq était considéré comme citadin, aujourd'hui au moins un sur trois. Au Gabon, en Côted'Ivoire et au Cameroun, par exemple, respectivement $75 \%, 50 \%$ et $50 \%$ de la population vivent en zones urbaines.

Associées à des conditions de stabilité atmosphérique marquées et des vents faibles, notamment en début de soirée, ces émissions conduisent à une qualité de l'air très médiocre, qui risque à moyen terme de poser de sérieux problèmes de santé publique. Une trop grande concentration dans l'atmosphère en composés soufrés et organiques ainsi qu'en métaux lourds peut avoir, en effet, des effets néfastes sur les systèmes respiratoire et cardiovasculaire.

\section{Des premières} mesures

Des études préliminaires menées par des chercheurs du Laboratoire d'aérologie et de différents laboratoires africains sur la pollution atmosphérique dans plusieurs grandes villes d'Afrique ont confirmé l'importance du problème. Nous pouvons citer, par exemple, 


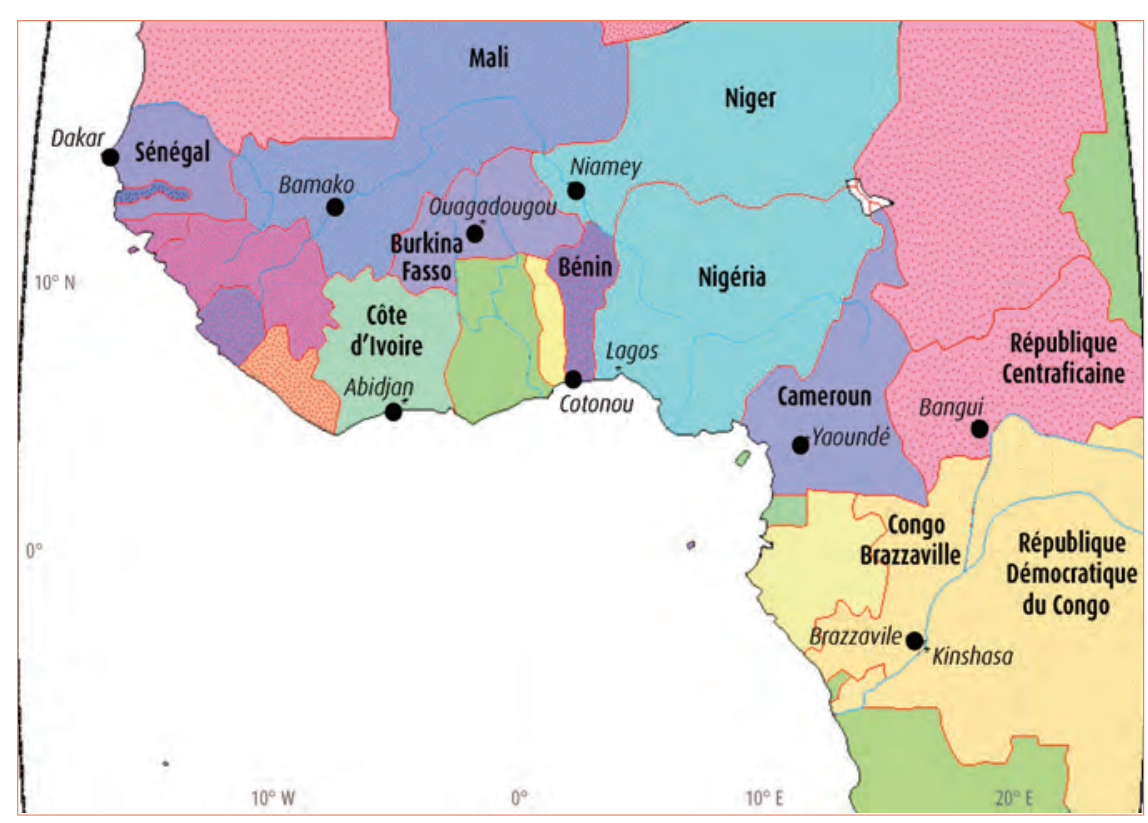

Figure 2 - Carte des capitales étudiées en Afrique de l'Ouest centrale : Cotonou (Amma) ; Niamey, Ouagadougou, Bangui, Brazzaville, Yaoundé, Abidjan (test Polca) ; Dakar et Bamako (test Polca et programme Polca).

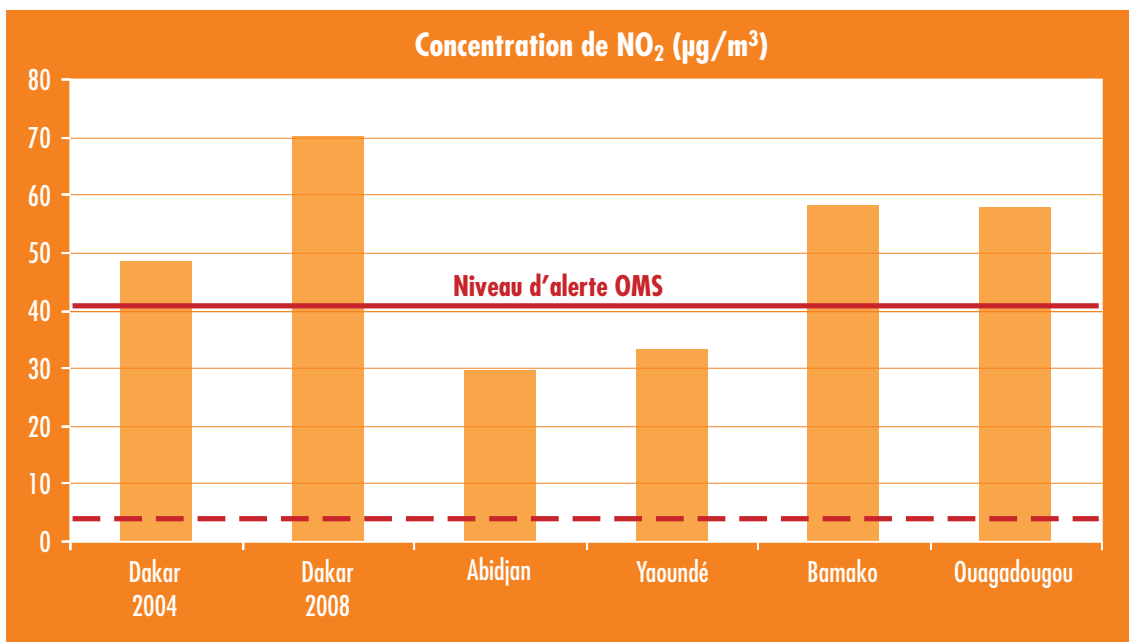

Figure 3 - Concentrations moyennes mensuelles de $\mathrm{NO}_{2}\left(\mu \mathrm{g} / \mathrm{m}^{3}\right)$ de cinq capitales d'Afrique de l'Ouest et centrale mesurées lors du projet test Polca en 2004 et en saison sèche en 2008 à Dakar dans le cadre Polca. La ligne rouge indique le seuil d'alerte annuel de l'Organisation mondiale de la santé $\left(40 \mu \mathrm{g} / \mathrm{m}^{3}\right)$ et le pointillé le niveau moyen mesuré sur les écosystèmes ruraux des sites africains du service d'observation Idaf (Adon et al., 2010 ; Yoboué, communication personnelle).

l'expérience-test Polca1 (Yoboué, communication personnelle) qui s'est déroulée en février-mars 2004 dans huit capitales africaines (figure 2) : Abidjan, Dakar, Bamako, Niamey, Ouagadougou, Bangui, Brazzaville et Yaoundé. Les concentrations atmosphériques de $\mathrm{SO}_{2}, \mathrm{HNO}_{3}, \mathrm{NO}_{2}, \mathrm{NH}_{3}$ et $\mathrm{O}_{3}$ représentatives de la saison sèche ont été mesurées. La figure 3 présente les concentrations de dioxyde d'azote en $\mu \mathrm{g} / \mathrm{m}^{3}$, mesurées en février-mars 2004 à Dakar, Abidjan, Yaoundé, Ouagadougou et Bamako, et la concentration moyenne mesurée en saison sèche en 2008 à Dakar dans le cadre du programme Polca2 (voir paragraphe suivant). Les concentrations mesurées de $\mathrm{NO}_{2}$, qui est un puissant d'aérosols de pollution à Cotonou (capitale du Bénin), en mai 2005. Des niveaux élevés de concentrations en carbone suie avec des pics minute de l'ordre de $86 \mu / \mathrm{m}^{3}$ ont été relevés, valeurs supérieures aux concentrations des grandes villes européennes (figure 4a). La dégradation de la qualité de l'air observée dans les capitales d'Afrique de l'Ouest s'accompagne, comme attendu, d'effets sur la santé publique. Des études pionnières réalisées par l'équipe du professeur Amadou Diouf, toxicologue à l'université de Dakar, ont montré une corrélation entre densité du trafic automobile et fréquence des affections bronchopulmonaires chez l'enfant, (variant de $22 \%$ à $36 \%$ suivant les sites) et chez les agents de police chargés de la circulation automobile avec une prédominance d'apparition d'asthme, rhume et broncho-pneumopathie (Diouf et al., 2003).

\section{Le programme Polca}

Dans ce contexte, nous avons initié le programme Polca (Pollution des capitales africaines) en collaboration avec nos collègues des universités africaines afin d'étudier la pollution chimique atmosphérique de deux capitales africaines (Bamako au Mali et Dakar au Sénégal) et son impact sur la santé humaine, notamment sur l'appareil respiratoire. Cette étude offre un cadre particulièrement favorable pour répondre aux questions scientifiques suivantes :

- quels sont les niveaux d'exposition annuels, saisonniers (particules, gaz) des populations à la pollution urbaine? - quelles sont les caractéristiques physico-chimiques de cette pollution? - quels sont les effets biologiques/ toxicologiques de cette exposition en termes de mécanismes et processus (stress oxydant, réactions immunologiques et inflammatoires...) ?

- quels sont les liens entre les données d'exposition et les affections respiratoires relevées dans les centres de santé (études épidémiologiques)? annuel fixé par l'Organisation mondiale de la santé $\left(40 \mu \mathrm{g} / \mathrm{m}^{3}\right)$. Le niveau de concentration moyen annuel de $\mathrm{NO}_{2}$ mesuré sur les sites ruraux du réseau de mesure Idaf (www.obs-mip.fr/IDAF/) indique des concentrations bien inférieures, comprises entre 0 et $6 \mu \mathrm{g} / \mathrm{m}^{3}$ (Adon et al., 2010).

Alertés par ces niveaux de pollution, et dans le cadre du programme Amma, nous avons effectué des mesures

\section{Stratégie expérimentale}

Notre stratégie expérimentale est fondée sur des études à court terme (une année), déployées au centre-ville de Bamako (Mali) et de Dakar (Sénégal), sur des carrefours de circulation représentatifs des activités urbaines d'une ville africaine (présence de policiers en 
service, vendeurs à l'étal et forte densité de population). Des mesures, à fréquence hebdomadaire, permettent la caractérisation chimique de l'aérosol atmosphérique (organiques, inorganiques, métaux). Des mesures bimensuelles des concentrations gazeuses $\left(\mathrm{NO}_{2}, \mathrm{O}_{3}, \mathrm{SO}_{2} \ldots\right)$ par capteurs passifs y sont associées. De plus, des campagnes in situ intensives en saison sèche (lien chimie-taille, solubilité de l'aérosol, analyseurs actifs de gaz...) ont eu lieu sur les sites de Bamako en janvier 2009 et de Dakar en décembre 2009. En parallèle à ces mesures, une stratégie expérimentale de mesures de santé a été développée par nos collègues toxicologues et médecins. Des cohortes spécifiques de personnes, sous influence de la pollution de ces carrefours routiers, ont été identifiées et sont soumises à des prélèvements ou tests biologiques (prises de sang). Des analyses toxicologiques in vitro sur des cellules bronchiques soumises à l'aérosol prélevé sur les sites ont été réalisées afin de mesurer le caractère inflammatoire de l'aérosol de combustion. Enfin, des données épidémiologiques sur les différentes affections respiratoires (asthme, broncho-pneumopathie chronique obstructive, emphysème...), relevées dans les structures hospitalières ou centres de santé proches de nos sites de mesures, seront recueillies et analysées. L'analyse de ces résultats est en cours dans le cadre de plusieurs travaux (thèses, master...) dans les universités de Toulouse, Paris, Abidjan (Côte-d'Ivoire), Bamako (Mali), Yaoundé (Cameroun) et Dakar (Sénégal).

\section{Quelques résultats préliminaires}

La figure 4a montre les premiers résultats de moyennes mensuelles de carbone suie (BC) obtenus à Bamako (Mali) et à Dakar (Sénégal), en avril et mai 2008 respectivement en partenariat avec A. Ndiaye et B. Diop. Les niveaux de pollution en carbone suie sont exceptionnels. Ils sont cinq à dix fois supérieurs à ceux de Paris (Cachier, communication personnelle dans le cadre du programme Caramel) et deux fois supérieurs à ceux mesurés au centre de Pékin (Guinot et al., 2007). Les variations de BC en cours de journée (figure $4 \mathrm{~b}$ pour Bamako) sont semblables sur les deux sites pour la période étudiée : on observe un pic de pollution le matin, un autre très important en soirée et des concentrations minimales la nuit. On peut

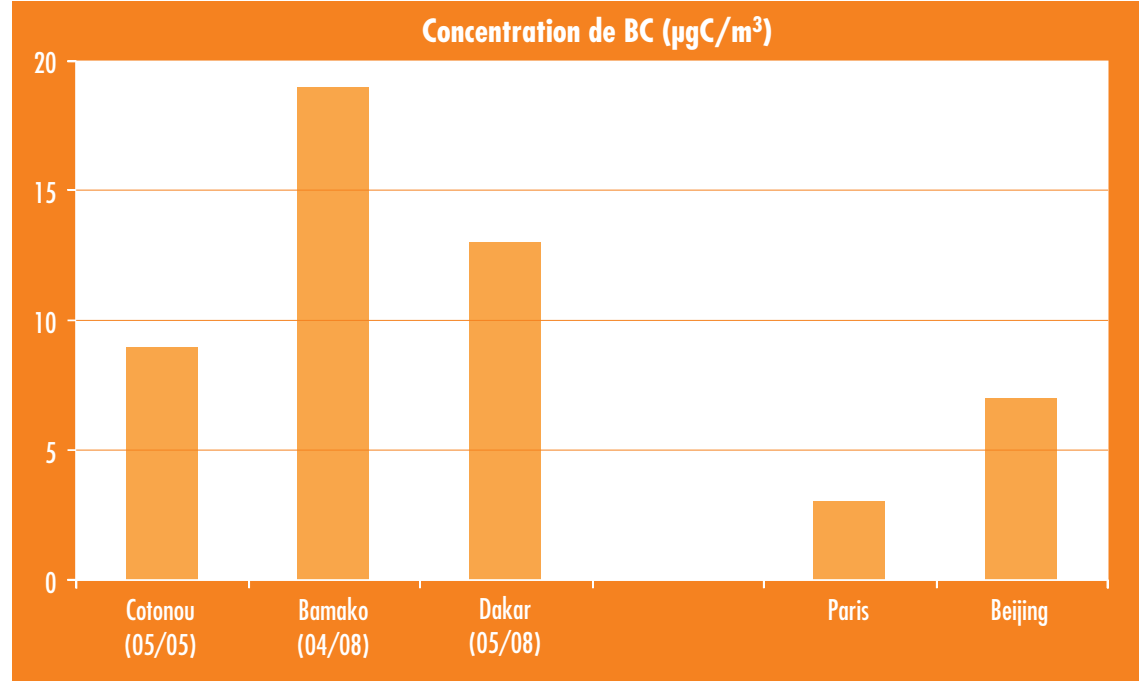

Figure $4 \mathrm{a}$ - Concentrations de carbone suie $(\mathrm{BC})$ en $\mu \mathrm{gC} / \mathrm{m}^{3}$, mesurées dans le cadre d'Amma à Cotonou et de Polca à Dakar et à Bamako. Les données (moyennes annuelles) de Paris (Cachier, communication personnelle) et de Beijing (Guinot et al., 2007) sont mentionnées pour comparaison.

remarquer une influence marquée de la réduction d'activité pendant les weekends (par exemple le 20/04) sur les concentrations de $\mathrm{BC}$. On peut noter enfin sur la figure $4 \mathrm{~b}$ que la concentration de $\mathrm{BC}$ à la longueur d'onde $370 \mathrm{~nm}$ est parfois supérieure à celle mesurée à $880 \mathrm{~nm}$. Cela révèle la présence importante d'un aérosol absorbant dans l'UV, de nature organique (HAP...). Selon les premiers résultats observés à Cotonou, Bamako et Dakar, ce phénomène est plus marqué en journée. Notons que la poursuite de la mesure en temps réel des concentrations de $\mathrm{BC}$ a permis de confirmer ces investigations à des échelles de temps plus longues, essentielles pour les applications santé (Doumbia et al., 2010).

\section{Programmes à plus long terme}

Polca est l'un des premiers programmes de recherche dédié à la quantification et la caractérisation de la pollution atmosphérique des capitales africaines, principalement liée aux sources de trafic routier, et à son impact sur la santé. D'autres campagnes de mesures sont nécessaires pour préciser la variabilité annuelle et saisonnière des émissions et des niveaux de pollution gazeuse et particulaire auxquels sont exposées les populations, incluant de nouvelles sources (combustion des déchets, industries, émissions des feux domestiques...) et de nouvelles capitales. L'ouverture de sites urbains à Bamako

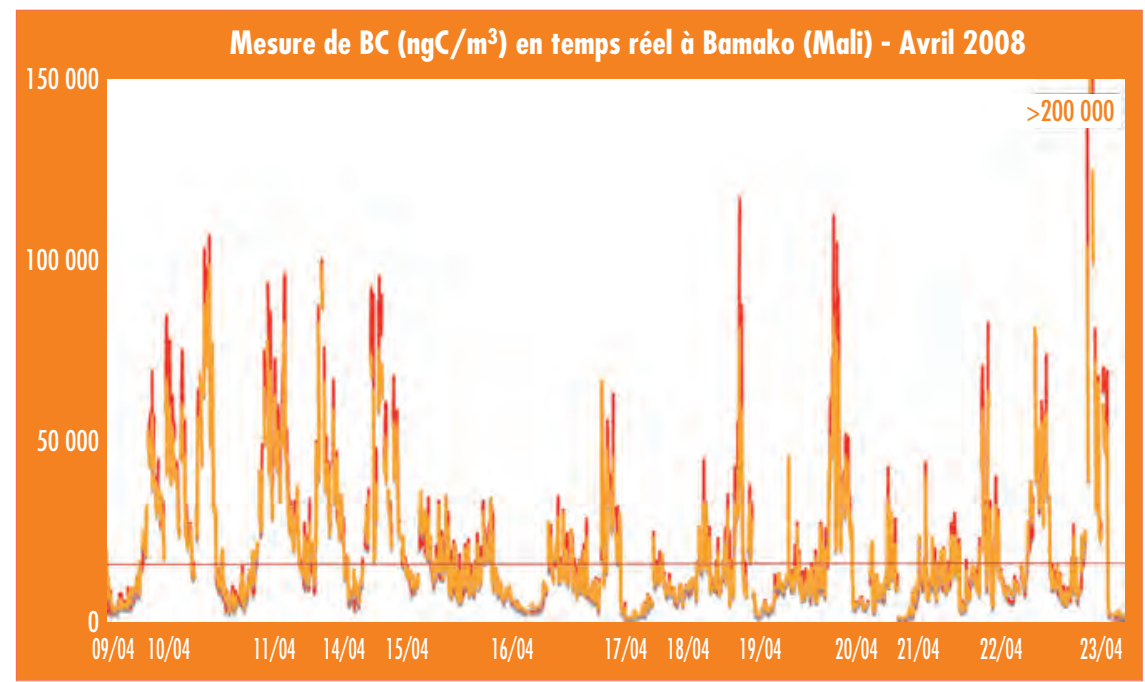

Figure $4 \mathrm{~b}$ - Mesures en temps réel des concentrations de carbone suie $(\mathrm{BC})$ à $880 \mathrm{~nm}$ (en rouge) et $370 \mathrm{~nm}$ (en orange) en $\mathrm{ngC} / \mathrm{m}^{3}$ à Bamako (Mali). La droite rouge représente la moyenne des concentrations de $\mathrm{BC}$ à $880 \mathrm{~nm}$. 
(Mali) et à Yaoundé (Cameroun) dans le cadre du réseau d'observation Idaf en Afrique, en lien avec des médecins et toxicologues, va permettre de poursuivre ces études et d'intégrer au mieux les aspects santé-environnement dans un contexte pluridisciplinaire et à long terme, indispensables aux études environnementales toxicologiques et épidémiologiques. Enfin, il est à noter que cette action, à présent partie prenante de l'axe environnement-santé-société de l'Observatoire Midi-Pyrénées (OMP) de Toulouse et du pôle de recherche et d'enseignement supérieur de l'université Paul-Sabatier de Toulouse, s'inscrit dans les objectifs scientifiques des programmes internationaux :

- IRD (Institut de recherche pour le développement) ;

- Debits (Deposition of Biogeochemically Important Trace Species) ; - Organisation météorologique mondiale (OMM) : surveillance de l'atmosphère globale ;
- IGBP/IGAC/Mégacités (International Geosphere Biosphere Programme/International Global Atmospheric Chemistry/Mégacités) ;

- Polca ;

- Amma2 ;

- GDRI - Arsaio (Groupement de recherche international - Atmospheric Research in Southern Africa and Indian Ocean).

\section{Bibliographie}

\footnotetext{
Adon M., C. Galy-Lacaux, C. Delon, V. Yoboué, K. Pienaar, C. Liousse, H. Al Ourabi, B. Diop, L. Dungall, L. Sigha, A. Akpo, J. P. Tathy, F. Lavenu, E. Gardrat : et P. Castera, 2010 : Long term measurements of sulfur dioxide, nitrogen dioxide, ammonia, nitric acid and ozone in Africa using passive samplers. Atmos. Chem. Phys., 10, 7467-7487.

: Assamoi E. et C. Liousse, 2010 : Focus on the impact of two-wheel vehicles on African combustion aerosols emissions. Atmos. Environ., 44, 3985-3996.

: Diouf A., G. Garçon et al., 2003 : Environmental lead exposure and its relationship to traffic density among Senegalese children: a pilot study. Human \& Experimental Toxicology, 22, 559-564.

Doumbia T., C. Liousse, C. Galy-Lacaux, A. Ndiaye, B. Diop, V. Yoboué, E. Gardrat, P. Castéra et L. Sigha, 2010 : Real-time black carbon measurements in West - Africa urban sites. Soumis à Atmos. Environ.

Guinot B., H. Cachier, J. Sciare, T. Yu et X. Wang, 2007 : Beijing aerosols: atmospheric interactions and new trends. J. Geophys. Res., 112, D14314, : DOl:10.1029/2006JD008195.
} 